\title{
NSCLC-Screening \\ Führen Angiogenesemarker im Atemkondensat zur Diagnose?
}

\author{
Gessner C et al. \\ Angiogenic markers \\ in breath condensate \\ identify non-small \\ lung cancer. \\ Lung Cancer. \\ 2010;68(2):177-84 \\ (Epub 2009, Aug 3).
}

\section{Hintergrund}

Derzeit gibt es kein verlässliches diagnostisches Verfahren, das sich zum Lungenkrebsscreening bei Risikopatienten (z.B. Rauchern) eignet.

\section{Fragestellung}

Die Studie untersuchte, ob sich mit Angiogenesemarkern im Atemkondensat ein Lungenkarzinom diagnostizieren lässt.

\begin{tabular}{|c|c|c|c|c|c|}
\hline Tabelle 1 & Messerg & ebnisse aus dem & emkondensa & & \\
\hline Patienten & Gesunde & Stabile COPD & AE-COPD & ndNSCLC & prNSCLC \\
\hline Anzahl & 12 & 15 & 15 & 17 & 15 \\
\hline IL-8 (pg/ml) & n.d. $(0,26)$ & n.d. $(0,29)$ & 6,5 & n.d. $(0,34)$ & n.d. $(1,12)$ \\
\hline $\begin{array}{l}\text { TNF-alpha } \\
\text { (pg/ml) }\end{array}$ & n.d. $(0,12)$ & n.d. $(0,16)$ & 5,16 & n.d. $(0,22)$ & n.d. $(0,41)$ \\
\hline VEGF (pg/ml) & 4,72 & 5,71 & 12,42 & 39,97 & 12,27 \\
\hline bFGF (pg/ml) & 5,40 & 6,99 & 12,92 & 82,33 & 12,79 \\
\hline $\begin{array}{l}\text { Angiogenin } \\
(\mathrm{pg} / \mathrm{ml})\end{array}$ & 3,03 & 4,20 & 7,51 & 68,76 & 6,15 \\
\hline
\end{tabular}

AE-COPD = akut exazerbierte COPD-Patienten; ndNSCLC = neu diagnostiziertes nicht kleinzelliges Lungenkarzinom; prNSCLC = nicht kleinzelliges Lungenkarzinom in partieller Remission; VEGF = vaskulärer endothelialer Wachstumsfaktor, bFGF = basischer Fibroblastenwachstumsfaktor; n.d. = unterhalb der Detektionsgrenze

\section{Eignungsnachweis steht noch aus Kommentar von Adrian Gillissen}

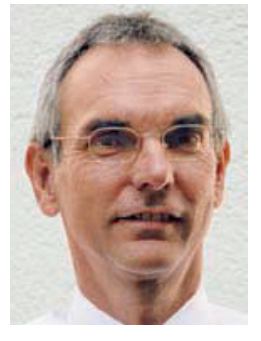

Prof. Dr. med. Adrian Gillissen, Robert-Koch-Klinik Leipzig

E-Mail: Adrian Gillissen@web.de achdem Ende der 1990er-Jahre und am Anfang des neuen Jahrtausends sehr viele Arbeitsgruppen mit dem Atemkondensat arbeiteten, ist es um diese Methode wieder sehr ruhig geworden. Gründe hierfür sind die fehlende Möglichkeit einer Standardisierung und die für die meisten Parameter sehr niedrigen, meist an der unteren Nachweisgrenze liegenden Substanz- und DNA-Konzentrationen [2]. In dieser Arbeit wurde die Kalibrierungskurve für die genannten Parameter nach unten justiert und bewegte sich damit außerhalb der Herstellerangaben. Es ergab sich eine hohe Sensitivität und Spezifität bei den genannten Patienten mit bekannter Diagnose. Ungeachtet dessen stehen die hier vorgestellten Daten im Widerspruch zur Studie von Dalaveris et al. [1]. Offenbar hängen die Ergebnisse aus

\section{Methodik}

In die Studie waren zwölf gesunde Nichtraucher, 17 Patienten mit einem neu diagnostizierten nicht kleinzelligen Lungentumor (ndNSCLC), 15 Patienten mit einem NSCLC in partieller oder kompletter Remission (prNSCLC) nach Chemotherapie, 15 Patienten mit stabiler COPD und 15 Patienten mit akut exazerbierter COPD (AE-COPD) eingeschlossen.

\section{Ergebnisse}

Der vaskuläre endotheliale Wachstumsfaktor (VEGF), der basische Fibroblastenwachstumsfaktor (bFGF) und Angiogenin waren beim ndNSCLC gegenüber anderen Gruppen im Atemkondensat signifikant bis um das Achtfache erhöht. Beim Tumor-Nekrose-Faktor-alpha (TNF-alpha) und bei Interleukin-8 (IL-8) zeigten sich dagegen keine signifikanten Unterschiede (Tab. 1).

Schlüsselwörter: Lungenkarzinom - Angiogenesemarker - Atemkondensat- NSCLC-Screening
Atemkondensatstudien stark von den jeweiligen Untersuchern $a b$, sodass sich die Quantifizierung von Angiogenesefaktoren auf keinen Fall als Screeninginstrument für Risikogruppen eignet. Erst der Nachweis einer hohen Sensitivität und Spezifität in einer multizentrischen Studie, die an nicht diagnostizierten Lungentumorpatienten in großer Anzahl durchgeführt werden müsste, könnte einen ersten Hinweis darauf geben, ob sich die Methodik überhaupt als Diagnostikum geschweige denn als Screeninginstrument eignet.

\footnotetext{
Literatur

1. Dalaveris E et al. Lung Cancer 2009;64:219-25

2. Horvath I et al. Eur Respir J 2005;26:523-48

3. Infante M et al. Am J RespirCrit Care Med 009;180:445-53
} 